\title{
An IoT Based Health Monitoring System to Tackle COVID-19 in a Contagious Ward of Hospital
}

\author{
Sk. Shariful Alam, Md. Shakibul Islam, Md. Mustahsin Farhan Chowdhury, Tanim Ahmed
}

\begin{abstract}
Regarding the highly contiguous novel coronavirus disease (COVID-19), it is unsafe for health professionals, being involved in a hospital isolation ward. The healthcare workers, stationed in a contagious ward provide relentless monitoring of specific health parameters of those patients. The rate of contagion proportionately depends on the time spent by the health workers in an isolation ward. This challenging task is fairly manageable by employing promising Internet-of-Things (IoT) based autonomous robots (i.e. smart bots) thus reducing the hazards of contamination to health workers. In this research paper, we introduce a smart bot that can periodically measure the health parameters of COVID-19 patients, for instance, body temperature, oxygen saturation levels, blood pressure, respiration rate, heart rate, blood glucose level, etc. The proposed smart bot will transfer from one patient to another in a structured pattern and autonomously collect, forward, store the data of the patients for further analysis. The goal of the work is to involve a significantly inferior number of health workers in contagious wards to control contagion, thus creating stress-free environs for health workers. Moreover, smart bots will offer health professionals to pay attention to non-COVID-19 patients and make things easier for regular health check-ups of individuals in need.
\end{abstract}

Keywords: Autonomous robot, Covid-19, RFID, RPPG.

\section{INTRODUCTION}

Novel Coronavirus disease 2019 (COVID-19) is greatly contagious caused by severe acute respiratory syndrome

Manuscript received on 27 July 2021 | Revised Manuscript received on 03 August 2021 | Manuscript Accepted on 15 August 2021 | Manuscript published on 30 August 2021.

* Correspondence Author

Sk. Shariful Alam*, AstroCeNT@Nicolaus Copernicus Astronomical Centre, Warsaw, Poland. Email: alamsh@camk.edu.pl

Md. Shakibul Islam, Student, Department of Electronics and Communication Engineering, Khulna University of Engineering \& Technology, Bangladesh. Email: islam1609027@stud.kuet.ac.bd

Md. Mustahsin Farhan Chowdhury, Pursuing, B.Sc. Degree, Department of Electronics and Communication Engineering, Khulna University of Engineering \& Technology, Bangladesh. Email: chowdhury1609028@stud.kuet.ac.bd

Tanim Ahmed, Pursuing, B.Sc. Degree, Department of Electronics and Communication Engineering, Khulna University of Engineering \& Technology, Bangladesh. Email: ahmed1609030@stud.kuet.ac.bd

(C) The Authors. Published by Lattice Science Publication (LSP). This is an open access article under the CC-BY-NC-ND license (http://creativecommons.org/licenses/by-nc-nd/4.0/)

Coronavirus 2 (SARS-CoV-2; formerly called 2019-nCoV) [1]. The average number of cases caused by a COVID-19 carrier is expressed as the reproduction number R_0. In the last century of the United Nations history, the Coronavirus is highly transmissible because of having an estimated value of R_0 between 1.50 and 3.50 [2]. As of 4th March 2021, there are about $116 \mathrm{M}$ cases recorded from which about $91 \mathrm{M}$ patients recovered, having about $2.57 \mathrm{M}$ deaths with active cases still tolling at about 21.7M [3], and the worst thing is that the numbers are increasing on a daily basis. So, the number of medical professionals needed are quite extensive, who are more likely to be infected. According to Public Health England, the staff who have face-to-face contact, or spending more than 15 minutes within 2 meters of an infected person have a high risk of being affected [4]. By maintaining proper hygiene, social distancing, and taking isolation measures the outbreak of COVID-19 can be controlled. It is contended that intelligent robotics and autonomous systems, as well as smart wearables, can play a positive role in order to facilitate prevention, containment, and mitigation of COVID-19 [2]. Therefore, to lessen the contact duration among the people, usage of automation technology is a viable solution. Moreover, autonomous systems can support the health workers to relieve the stress of managing COVID-19 patients which consequently facilitates the non-COVID-19 patients' treatment, as well as permits regular health check-ups for the people who are in need.

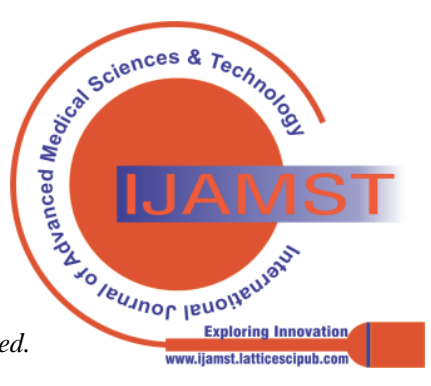




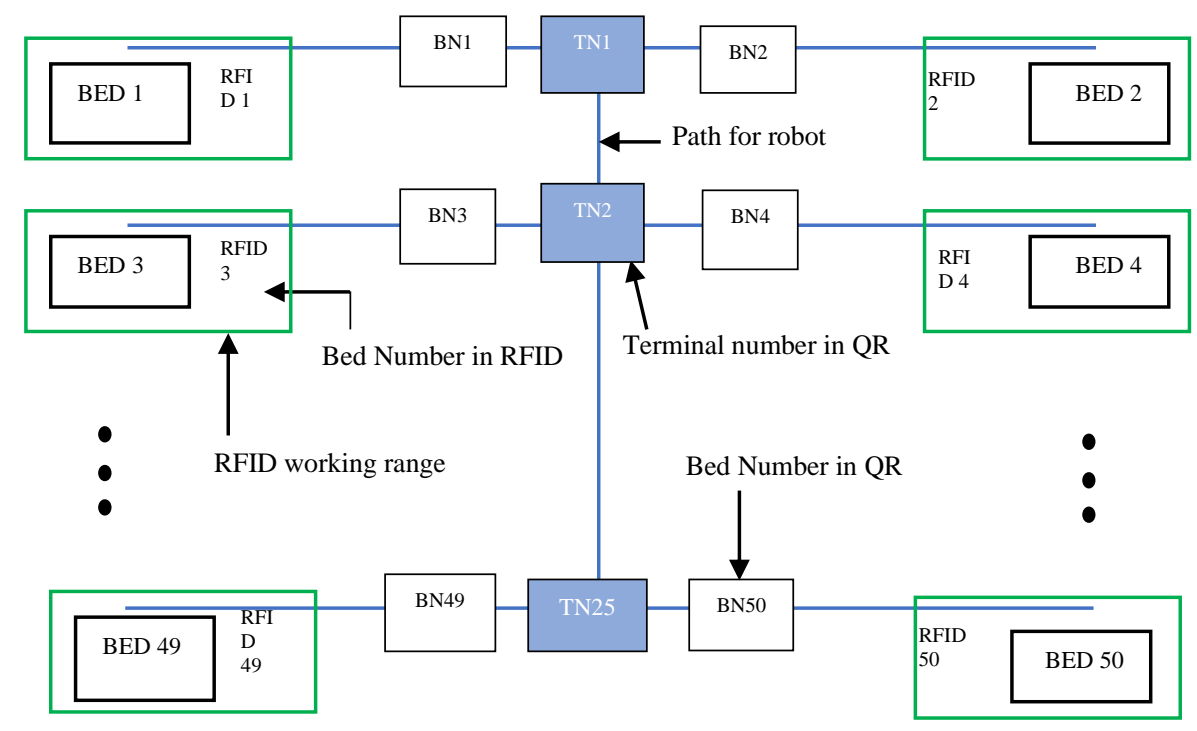

Fig. 1. Illustration of path mapping of an isolation ward.

To shrink the amount of time of the medical staff in an isolation ward, a smart bot is required. The smart bot moves from bed to bed following a predefined locus using the path mapping as presented in Figure 1. The patient as well as the bed is fixed within a certain area and the smart bot moves periodically from one patient to another and collects clinical data. Those data are forwarded to the server established at the outside of the isolation center for further dealing out and investigation. However, if an emergency situation occurs to a patient, smart bot has the flexibility to transfer to a particular bed, perform the task then resume the previous job. This feature is illustrated in Figure 2.

As fever is one of the most obvious symptoms of the COVID-19, the body temperature reading is compulsory. Since COVID-19 is a respiratory disease, the condition of the lungs, oxygen saturation levels, heart rate, and respiration rate monitoring of patients are important factors. According to research, patients with a medical background of having diabetes, or high blood pressure, or both might develop critical conditions [5]. In consequence, blood pressure and glucose level measurement are also important. All these measurements are taken in contactless methods to reduce the chances of contamination through equipment. All patient data is transmitted to the control server in real-time and stored in a spreadsheet for later diagnosis and post-processing.

For an interactive conversation between the doctor and patient, a video calling feature is enabled at the smart bot. An ultraviolet sterilization box is attached to sterilize equipment periodically. A protective cover is placed all over the robot to reduce the chances of contamination from it. A light source with intensity control is used to improve visibility in low light conditions. The robot can also supply prescribed medicines to the desired patients.

The goal is to involve a significant inferior number of health workers in contagious isolation wards whilst guaranteeing a proper treatment of the patients. The proposed system ensures the collections of the clinical parameters of COVID patients with fully autonomous functionality and it forwards, stores the collected data for further analysis. Further-more, the smart bot will have the capability of self-sanitization as it roams to patients' bed and also has the feature of providing medicine to the patients. Finally, the smart bot will return to its initial position after finishing the job period.

\section{RELATED WORK}

During the COVID-19 pandemic, researchers have focused on contactless patient monitoring systems more than ever as it is highly contagious. IoT-Edge Technologies can be used to find a person who is infected by COVID-19 to prevent an epidemic and block contagion. Besides, several advantages of smart automation systems as well as intelligent robotics have addressed in paper [1,2]. The infected person can be escorted to the hospital ward in a contactless method from the ambulance [6]. Contactless communication between health professionals and patients in such a pandemic is required and the possible solution is through the use of mobile robots [7]. The mobile robots are guided automatically by means of lines that are added to the patient's bed. Also, the medicine can be supplied in a similar manner [8]. In order to help the patients in their daily activities such as getting something or a reminder for taking medicines, a robot can be used which will work on audio input [9]. Things touched by a contagious patient are also contagious and need to be disposed of in order to diminish contamination and this kind of wastage can also be removed by autonomous robots. Patients are usually hospitalized and some are home quarantined, and their condition is monitored through exploiting wearable sensors whether their symptoms degrade or not [8-10]. To promote contactless action with the sensor as like as IR thermometer can be casted-off rather than a conventional way of measuring body temperature by means of mercury thermometer. Likewise, PPG is a non-invasive technology that can be used to measure different health parameters such as heart rate and oxygen saturation levels in the human body [11,12]. This kind of approach might increase the chances of contamination.

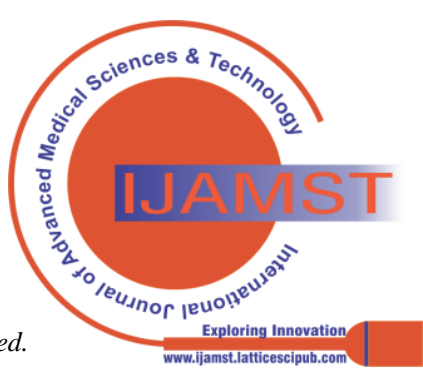




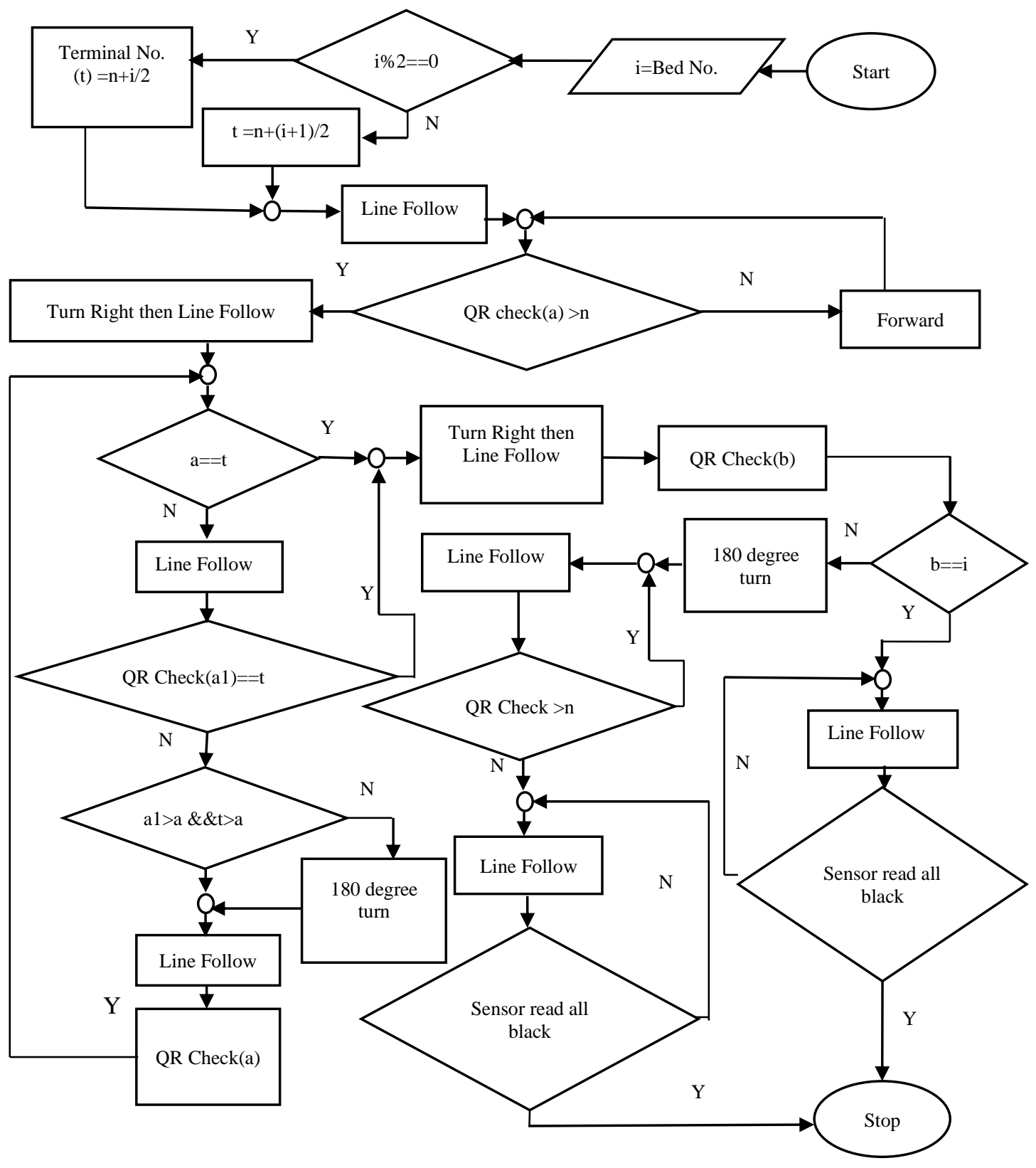

Fig. 2. Proposed maneuvering algorithm of the smart autobot.

So, to eliminate this risk, the rPPG technique is used $[13,14]$. Continuous glucose monitoring using an IoT-based system may reduce the opportunity of contamination, but it requires dedicated devices for every patient which will increase the cost [15]. In general, contact-based methods are used to measure respiration rate, but in order to reduce contamination, analyzing the thermal image from a thermal camera can be a good choice to monitor Glucose level and respiration rate without physical contact [16-18].

The main contributions of this paper are: a) the modular architecture of an IoT based autonomous robot has been suggested, which ensures the collection of clinical parameters from COVID-19 patients where the collected data is stored and forwarded for further analysis; $b$ ) an idea of how the smart bot moves in a contagious ward following a specific path; c) this paper also provides flowcharts and proper algorithms for each building block of the proposed smart bot; d) the integration of sensors that responsible for data processing and transmission is presented in this research work.
This paper is organized as follows: after this section, the following sections illustrate the design criteria, the working principle of the autonomous smart bot and integration of various sensors to shape the model and finally the conclusions.

\section{METHODOLOGY}

This section is based on the smart bot performing certain tasks following the path mapping as shown in Figure 1 taking bed numbers to be $n$ in total. This section is organized in three sub-sections named Maneuvering, Health Check-ups, and Data Processing and Communications. Those are discussed as in the following:

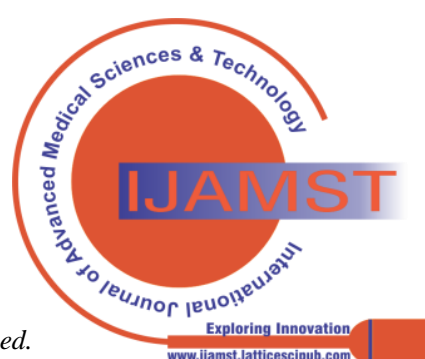




\section{A. Maneuvering Section}

This section consists of the design algorithm as well as architecture of the smart bot and how the smart bot perform is shown in Figure 2, Figure 3 and Figure 4.

\section{1) Mechanical Structure}

The smart bot is operated with four-wheels. A hollow pipe is attached to the robot with two arms with one having a three (03) degree of freedom (DoF) with a stethoscope attached to it and another having a two (02) DoF with a linear slider with which a camera and a flashlight is attached. The first one is used for better positioning regards to measuring the condition of the lungs and the latter is used for the proper focusing of the face. Since the robot is going to move using a line following algorithm it requires infrared sensors, so a QTR-8RC reflectance sensor array is attached to the base of the smart bot at an optimized height above the ground. A QR code scanner is required to trace both the bed number and the terminal number by scanning the QR code as shown in Figure 1. In addition, two sonar sensors are used in front and backside of the robot to avoid collision with objects.

The robot has worn a custom-made PPE while staying in the isolation ward and an ultraviolet ray is used to sanitize the equipment such as a camera and stethoscope to reduce chances of contamination of the patient from the robot. Moreover, an LDR sensor is present so that when the lighting is low, a light turns on automatically.

\section{2) Working Principles}

A contagious ward consisting of a certain number of beds that are sectioned as shown in Figure 1. To distinguish the

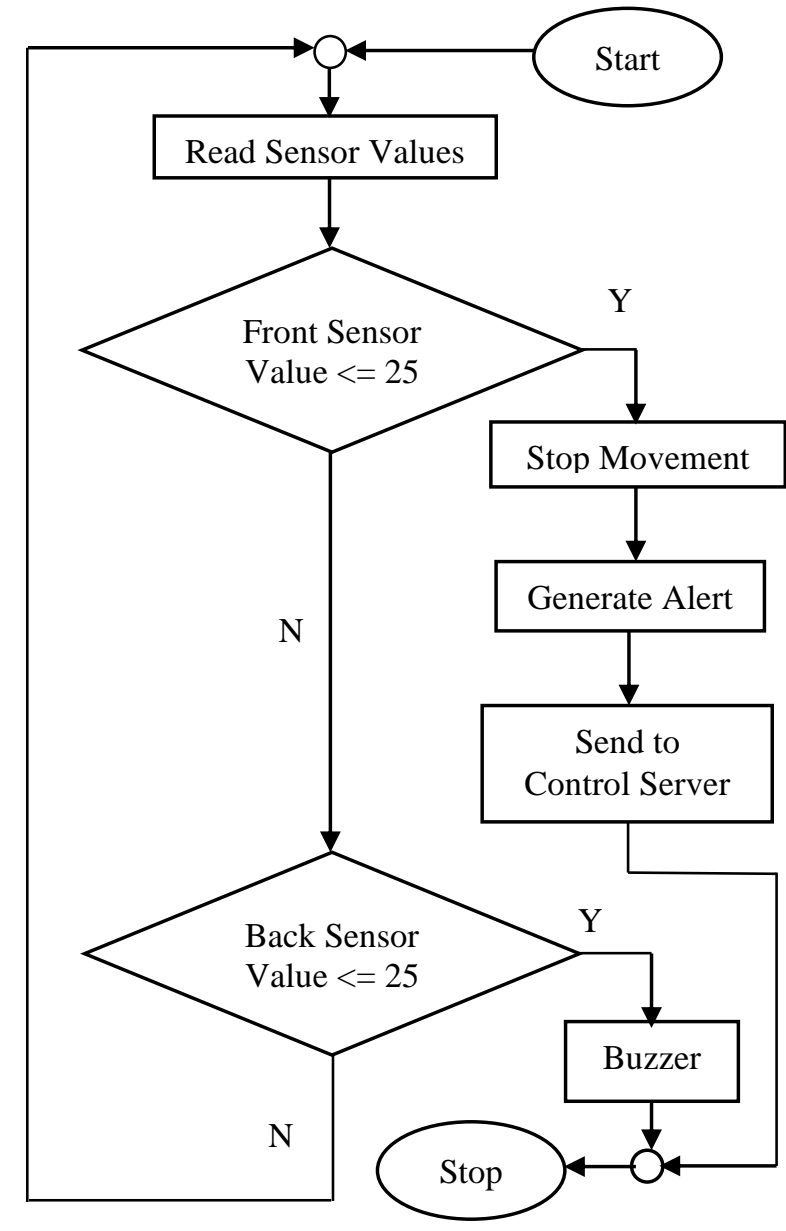

Fig. 3. Proposed algorithm for obstacle avoidance.

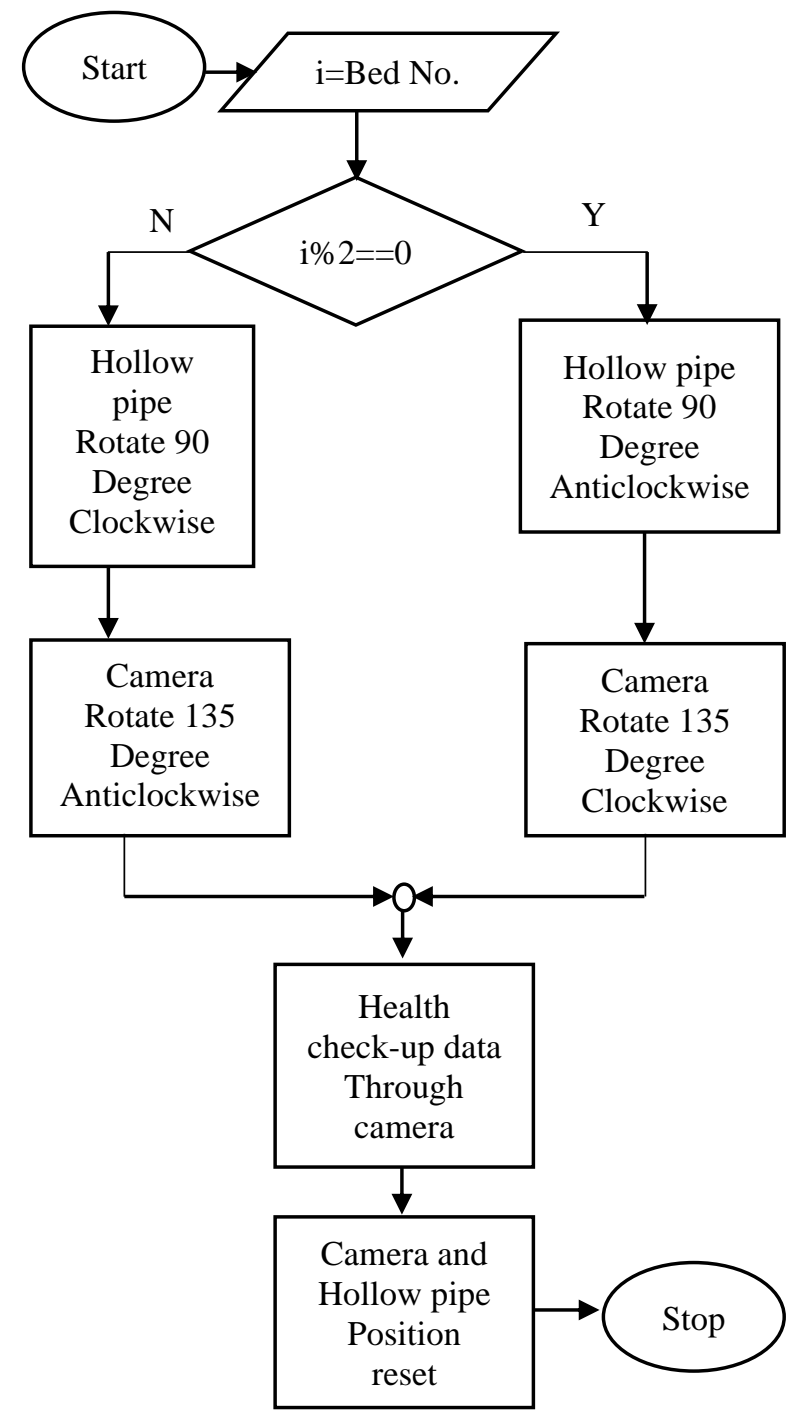

Fig. 4. Flowchart for camera movement of the smart autobot.

terminals from the beds, QR codes are used in every cross-section and for every bed. The cross-sections and the bed numbers are denoted by TN\#(number) and BN\#(number) respectively as shown in Figure 1. The robot uses the algorithm, as shown in Figure 2, to move to each bed in the ward where the total bed number is considered to be ' $n$ ' and the terminal number is denoted by greater than ' $n$ '. If the

robot gets derailed in any case, an alert message is sent to the control server.

Inside the isolation ward, there might come a situation where some objects might come in the way of the robot. To avoid collision an obstacle detecting mechanism is implemented using sonar sensors situated in the front and back end of the robot [19]. The collision avoidance algorithm is addressed in Figure 3. A threshold value of $25 \mathrm{~cm}$ is set for detecting objects for both front and back-end sensors. In case of the front and back sensor, if an object is detected at a distance less than $25 \mathrm{~cm}$, the robot stops and an alert is sent to the control server and a buzzer is activated to alert any visually impaired or unwary person who might collide with the robot.

Published By:

Lattice Science Publication

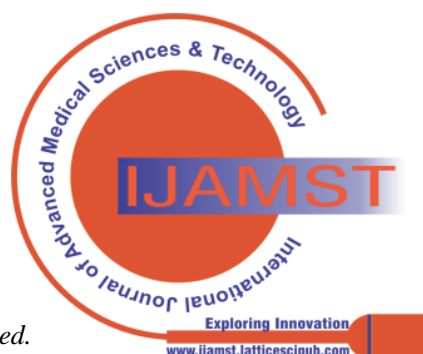


If none of the cases happen the robot continues to follow the line. When the robot reaches a bed the algorithm as shown in Figure 4 initiates. The camera system is attached to a linear slider to adjust the camera system to focus the patient's face using face detection. Clockwise and anticlockwise rotation for both hollow pipe and camera is used to secure accurate positioning as well as to avoid flipping of the video frame.

\section{B. Health Check-up}

The smart bot collects various health parameters of a patient after placing the camera in the correct position and being connected to the server through the RFID tag. An IR thermometer can be used to check body temperature remotely [11] while remote Photoplethysmography (rPPG) is used for measuring the Heart rate, oxygen saturation levels, and Blood pressure [20-22]. Thermal camera enabling thermal image processing feature can be employed to measure respiration rate and blood glucose levels [17,18]. A modified stethoscope is used to check the condition of the lungs and the audio signal is sent to the control server. The state-of-art approaches for measuring the specified health parameters are summarized as in the following.

\section{1) Remote Photoplethysmography (rPPG)}

The facial skin color along with the heart rate and Oxygen saturation (SpO2) can be monitored using the remote Photoplethysmography (rPPG) technology. In order to have an estimation of Oxygen saturation (SpO2), Blood pressure, and heart rate, the method processes video captured from a camera in real-time to attain the Photoplethysmography signal which detects changes in some regions of the face in cardiovascular tissue. The specific regions of the face are captured using the region of interest (ROI) detection. The regions are mainly concentrated on the forehead and cheeks, but more regions are included as some of them might be covered [21]. Then a set of tracking points are extracted from the central ROI. Therefore, the parameter's value is generated

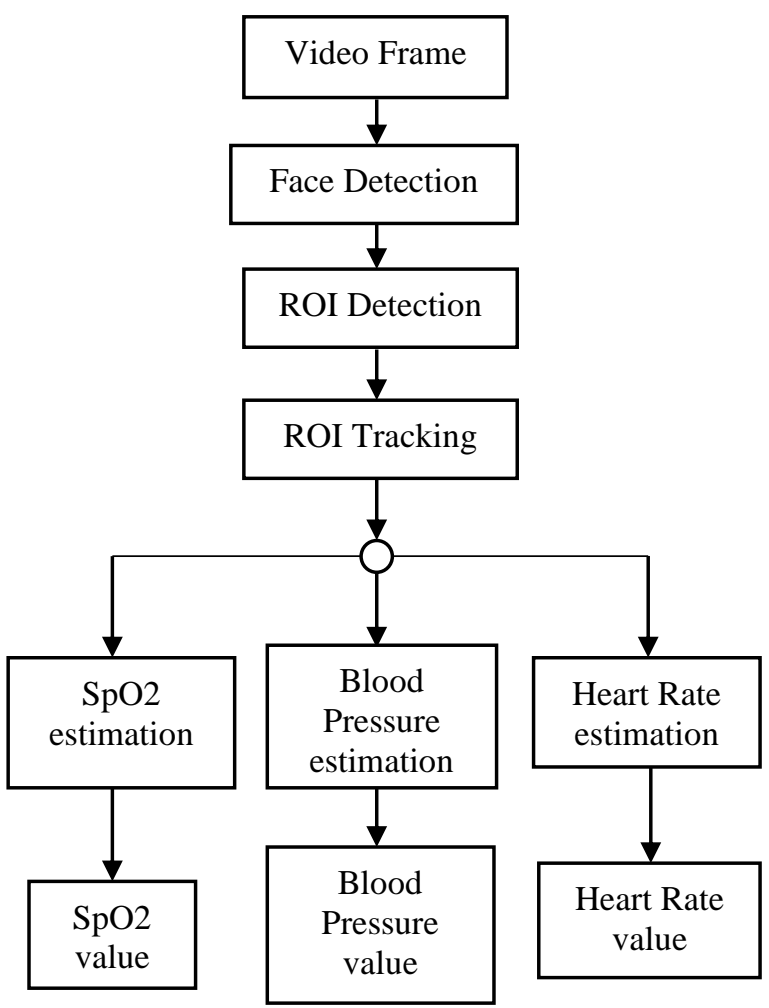

Fig. 5. Block Diagram for Blood Pressure, Oxygen saturation $\left(\mathrm{SpO}_{2}\right)$ and Heart rate measurement. by exploring different methods and this is exemplified in Figure 5.

\section{2) Thermal Image Processing}

Glucose level (i.e., blood sugar level) and respiration rate are obtained using thermal image processing of the human body which is shown in Figure 6. The parameters can be extracted from the image captured by the thermal camera using the 'Classification' algorithm. After capturing the thermal image, pre-processing is used to convert the thermal

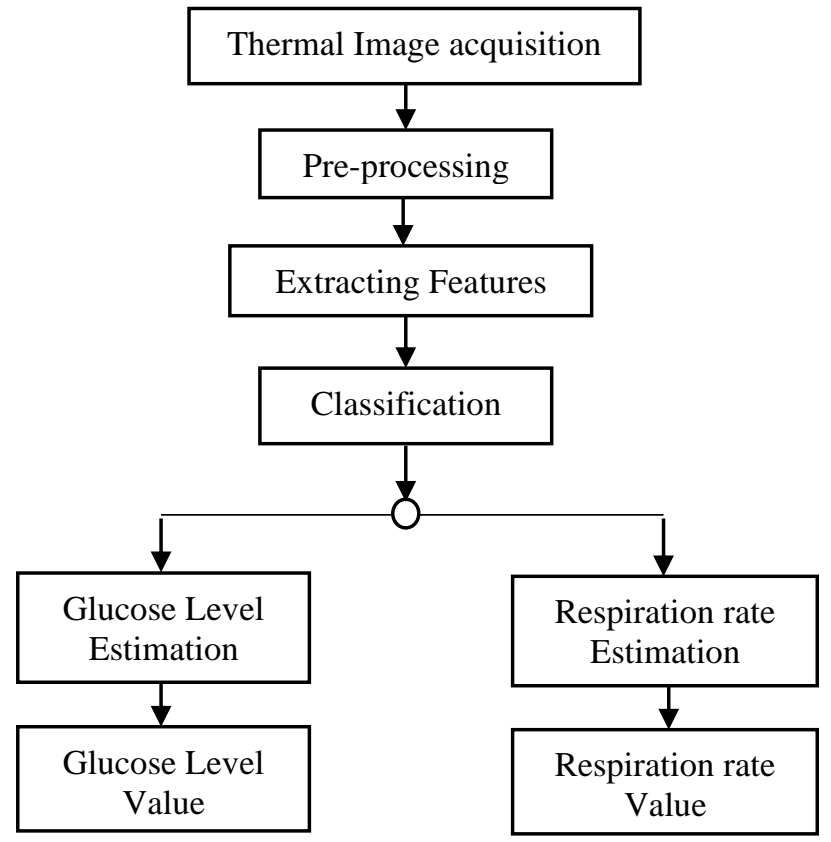

Fig. 6. Block Diagram for Glucose level and Respiration rate measurement.

image to a grayscale image. Then the features are extracted by comparing temperature variations of the lateral surfaces of the selected region. After selecting the required images some regions of interest (ROI) are identified from the acquired images for analyzing. After comparing these images, the approximate glucose level and respiration rate can be detected [17].

\section{3) Infrared Thermometer}

The infrared thermometer is used to measure the body temperature of a patient in a contactless method. An infrared thermometer module is used which consists of thermocouple sensors that convert the heat energy to electric voltage and a micro-controller unit that generates the temperature value for a respective voltage. The temperature value is then sent to the control server through the robot. The process is shown in Figure 7.

\section{4) Modified Stethoscope}

A modified stethoscope can be used to check the condition of the lungs which is one of the important parameters for COVID-19 patients. It consists of a microphone-like transducer that converts the air vibration of the stethoscope to an analog audio signal.

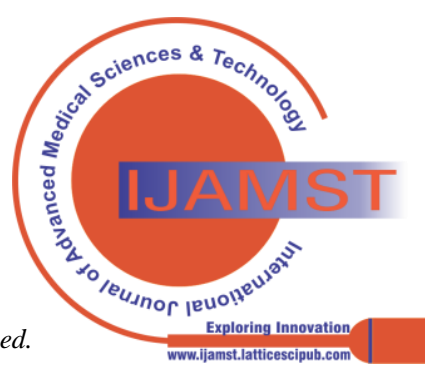


After passing through a low noise amplifier, the signal is converted to digital output via standard A/D converter, and then it is forwarded to the control server through the robot. The process is shown in Figure 8. On the contrary, in the user-end, a D/A converter converts the digital output to the audio signal again and the doctor/health worker can diagnose the condition of the lungs of the patient.

\section{Data Processing and Communication}

This section incorporates how the collected data is being transferred from the smart bot to the server and finally to medical professionals.

\section{1) Communication and Data Transfer}

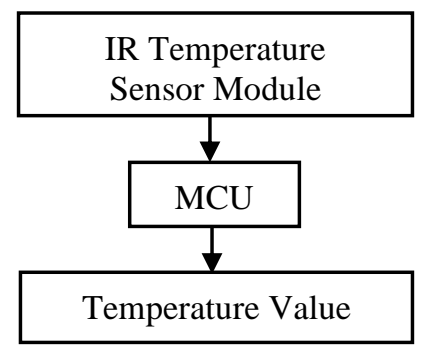

Fig. 7. Block Diagram for IR temperature measurement.

An RFID tag is chosen for the smart bot to indicate from what bed number the data is being collected and WLAN is used to send data to the server. The data is collected for each patient who is assigned to a particular bed. The process is shown in Figure 9. An RFID reader is attached to the raspberry pi module which allows the robot to scan the RFID tags containing bed numbers. When the robot first crosses the RFID tag, a signal is sent to the server that the health data of a particular bed is being recorded. The recorded data is sent to the server which is then formulated in an orderly manner for every patient according to the bed number.

\section{2) Control Server}

A control server is created which is able to communicate with both the robot and the hospital website at the same time. As the robot first scans an RFID tag, a message is sent to the control server to collect health check-up data for that particular bed with the matching patient. The collected data are organized in a spreadsheet consisting of the patient info with their corresponding health check-up data. Besides, the

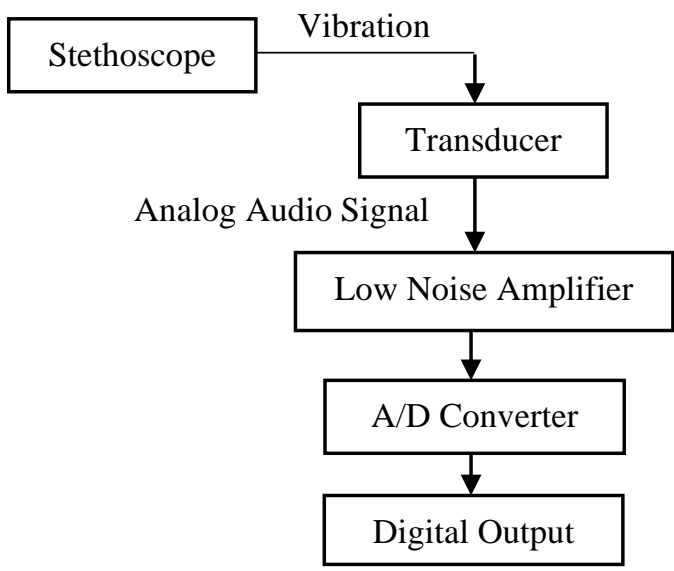

Fig. 8. Block Diagram for Lung condition measurement.

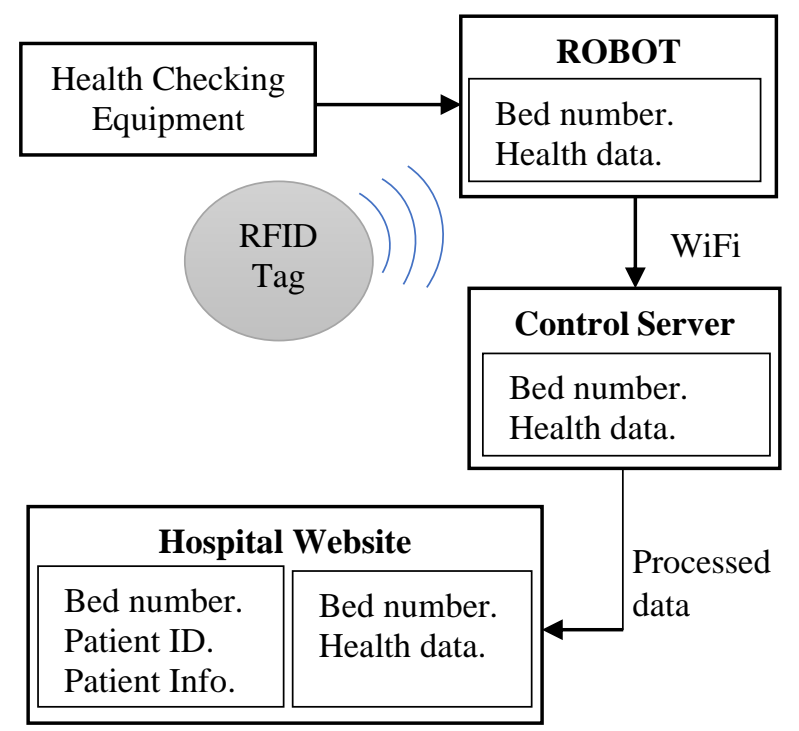

Fig. 9. Data processing and communication of the robot.

server also shows CCTV footage of the ward which allows the operator to inspect a patient's condition. If such a patient's condition degrades, the operator interrupts the robot's predefined path and reroute it towards the bed number of the patient with an emergence. Moreover, smart bot has video calling feature (when necessary) enabled through Wireless Local Area Network (WLAN) to establish a communication path between a medical professional and a patient from the isolation ward. Real-time diagnostic report of the bot's components which includes battery level, condition of the sensors and motors is sent to the control server as well as to the manufacturer's maintenance server via internet to enable remote monitoring and to generate alert if any malfunction occurs.

\section{3) Hospital Website}

Processed data from the control server is sent to the hospital website which keeps a record of the patient's health condition. The hospital website displays patient ID and short medical history data of a particular patient associated with a precise bed number. The hospital website encompasses medical history data of the patients that can only be accessed by health professionals or doctors on duty and diagnoses accordingly.

\section{RESULT AND DISCUSSION}

We have proposed various key modules in several figures of this paper for the smart Autobot while a lot of learning is yet to be carried out. We tried to implement the modified stethoscope to access the bronchi acoustical signals of a patient from a distant position. Vesicular breath sounds are low amplitude signals generated from the lung fields. Vesicular sounds are normally heard throughout inspiration, sound intensity faded rapidly for the period of expiration. Usually, inspiration is approximately three (03) times the length of expiration.

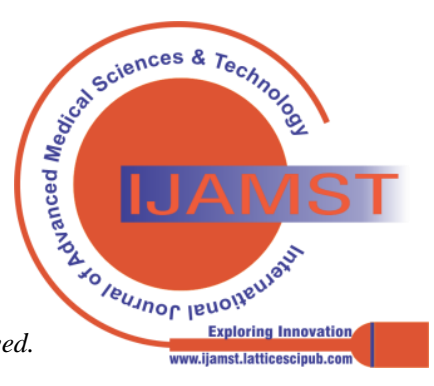


In this context, the analog signal of the lung can be captured by the Autobot module and then is converted to a digital signal. This signal is then transferred to a distant position where a health professional may check the vesicular breath sound (after converting from digital to an analog form of signal) and may decide for the patient. Fig. 10 provides a timing representation of vesicular breath sound generated through exploiting the proposed module in Fig.08. The time-varying nature of breath sound provides a good result which looks like the original signal captured by a precise stethoscope [23]. In the future, each of the proposed modules of the smart Autobot will be implemented and standardized to arrange the complete Autobot system which gives relief to the health professional from contagious diseases like Covid-19.

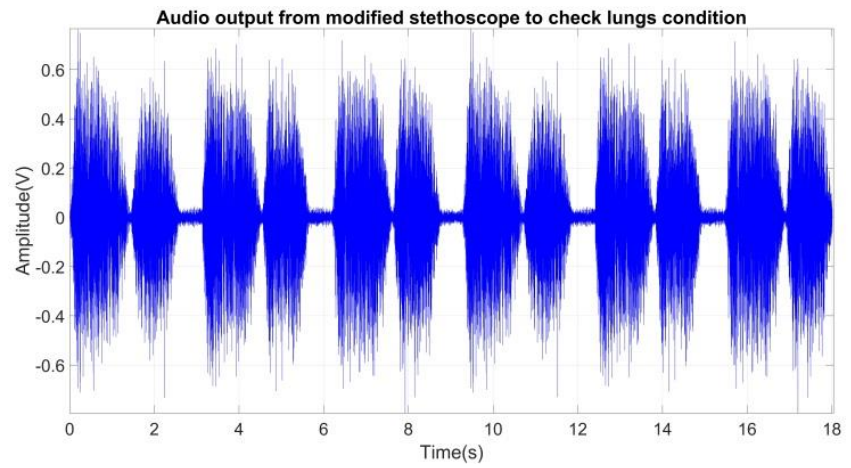

Fig.10. Illustration of Audio Signal captured from Fig.08

\section{CONCLUSION}

The incredible speed of the contagion of the COVID-19 pandemic has set under stress to the health workers to maintain a standard professional attitude. This stress may work as a promoter to apt discussions about interdisciplinary research, and may incorporate advanced applications of automation technology; go back over the arrangements of how the healthcare system is carried out. The proposed smart bot will explore the interdisciplinary dialog, as a result, practical, feasible, and favorable solutions will possibly be materialized to tackle effectively the present emergencies and the crisis yet to come.

The proposed smart bot can tackle contagious diseases efficiently in particular during the pandemic of COVID-19, the global crisis in healthcare, social, economic ground. It will be able to perform certain routine healthcare jobs that are being performed by medical staffs whilst maintaining proper hygiene. As a result, this approach will significantly decrease the probabilities of contamination as well as minimize the number of medical personnel involved in the COVID-19 ward. All the health data will be conserved in the hospital database for every patient and can be accessed anytime by the authorized staff. Limited number of resources (i.e. few sensors) are required and typical communication protocols (IR and WLAN) are used to build the smart bot which has made the proposed system simple to design. Besides, the health data will be measured for every patient, and that no dedicated device for each patient is required, hence the cost is reduced. Moreover, the limited number of resources and standard communications point toward less cost thus made the proposed smart bot cost-efficient. The major advantage of the proposed smart bot is to reduce the COVID-19 infected rate of medical professionals significantly as they involve less time in the isolation ward.

The proposed smart Autobot will offer assistance to the health professionals that lead them to pay attention to the non-COVID-19 patients and make things easier for regular health check-ups of individuals in need. Moreover, the smart bot can agree to smart monitoring of high-risk groups such as cardiac patients, patients with respiratory issues, etc. to remain in isolation at home while receiving rehabilitation therapy through a distance.

\section{ACKNOWLEDGMENT}

This research received no specific grant from any funding agency in the public, commercial, or not-for-profit sectors.

\section{REFERENCES}

1. M. U. Ashraf, A. Hannan, S. M. Cheema, Z. Ali, K. m. Jambi and A. Alofi, "Detection and Tracking Contagion using IoT-Edge Technologies: Confronting COVID-19 Pandemic," 2020 International Conference on Electrical, Communication, and Computer Engineering (ICECCE), 2020, pp. 1-6, doi: 10.1109/ICECCE49384.2020.9179284. [CrossRef]

2. M. Tavakoli, J. Carriere, A. Torabi, "Robotics, Smart Wearable Technologies, and Autonomous Intelligent Systems for Healthcare During the COVID-19 Pandemic: An Analysis of the State of the Art and Future Vision," Advanced Intelligent Systems, 2020, vol. 2, no. 7 , pp. 2000071, doi: https://doi.org/10.1002/aisy.202000071 [CrossRef]

3. Coronavirus Update (Live): 117,650,639 Cases and 2,608,987 Deaths from COVID-19 Virus Pandemic - Worldometer Available online: https://www.worldometers.info/coronavirus/?utm_campaign=homeAd UOA?Si\%23countries (accessed on 8 March 2021).

4. Coronavirus (COVID-19) Expert Interview: What Is Contact Tracing? Public Health Matters Available online: https://publichealthmatters.blog.gov.uk/2020/02/13/expert-interview-w hat-is-contact-tracing/ (accessed on 8 March 2021).

5. B. de Almeida-Pititto, P. M. Dualib, L. Zajdenverg, J. R. Dantas, F. D. de Souza, M. Rodacki, M. C. Bertoluci, "Severity and mortality of COVID 19 in patients with diabetes, hypertension and cardiovascular disease: a meta-analysis," Diabetol Metab Syndr 12, 2020, no. 75, doi https://doi.org/10.1186/s13098-020-00586-4. [CrossRef]

6. H. A. Hadi, "Line Follower Robot Arduino (using robot to control Patient bed who was infected with Covid-19 Virus)," 2020 4th International Symposium on Multidisciplinary Studies and Innovative Technologies (ISMSIT), 2020, pp. 1-3, doi: 10.1109/ISMSIT50672.2020.9254906. [CrossRef]

7. M. Cardona, F. Cortez, A. Palacios and K. Cerros, "Mobile Robots Application Against Covid-19 Pandemic," 2020 IEEE ANDESCON, 2020, pp. 1-5, doi: 10.1109/ANDESCON50619.2020.9272072. [CrossRef]

8. M. Antony, M. Parameswaran, N. Mathew, S. V.S, J. Joseph and C. M. Jacob, "Design and Implementation of Automatic Guided Vehicle for Hospital Application," 2020 5th International Conference on Communication and Electronics Systems (ICCES), 2020, pp. 1031-1036, doi: 10.1109/ICCES48766.2020.9137867. [CrossRef]

9. B. S. Pranathi, A. Nair, C. S. Anushree and T. S. Chandar, "Sahayantra A Patient Assistance Robot," 2020 11th International Conference on Computing, Communication and Networking Technologies (ICCCNT), 2020, pp. 1-6, doi: 10.1109/ICCCNT49239.2020.9225444. [CrossRef]

10. S. S. Kumar, "Emerging Technologies and Sensors That Can Be Used During the COVID-19 Pandemic," 2020 International Conference on UK-China Emerging Technologies (UCET), 2020, pp. 1-4, doi: 10.1109/UCET51115.2020.9205424. [CrossRef]

11. G. Long, "Design of a non-contact infrared thermometer," International Journal on Smart Sensing and Intelligent Systems, 2016, vol. 9, no. 2, pp. 1110-1129, doi: 10.21307/ijssis-2017-910. [CrossRef]

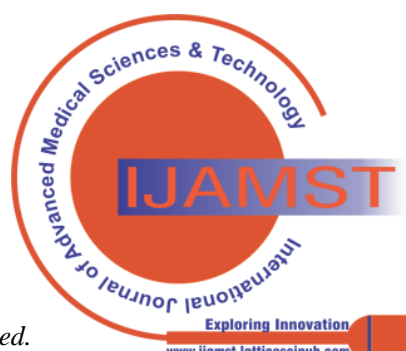


12. D. Castaneda, A. Esparza, M. Ghamari, C. Soltanpur, and H. Nazeran, "A Review on Wearable Photoplethysmography Sensors and Their Potential Future Applications in Health Care," Int J Biosens Bioelectron, 2018, vol. 4, pp. 195-202, doi:10.15406/ijbsbe.2018.04.00125. [CrossRef]

13. K. M. van der Kooij, M. Naber, “An Open-Source Remote Heart Rate Imaging Method with Practical Apparatus and Algorithms," Behav Res, 2019, vol. 51, pp. 2106-2119, doi:10.3758/s13428-019-01256-8. [CrossRef]

14. A. R. Guazzi, M. Villarroel, J. Jorge, J. Daly, M. C. Frise, P. A. Robbins, L. Tarassenko, "Non-Contact Measurement of Oxygen Saturation with an RGB Camera," Biomed. Opt. Express, 2015, vol. 6, pp. 3320, doi:10.1364/BOE.6.003320. [CrossRef]

15. T. N. Gia, M. AZzA Ali, I. B. Dhaou, A. M. Rahmani, T. Westerlund, P. Liljeberg, H. Tenhunen, "IoT-Based Continuous Glucose Monitoring System: A Feasibility Study," Procedia Computer Science, 2017, vol. 109, pp. 327-334, doi:10.1016/j.procs.2017.05.359. [CrossRef]

16. C. Massaroni, A. Nicolò, M. Sacchetti and E. Schena, "Contactless Methods for Measuring Respiratory Rate: A Review," in IEEE Sensors Journal, doi: 10.1109/JSEN.2020.3023486. [CrossRef]

17. M. S. Anand, M. Balamurugan, R. Shubathra, M. Ragavi, R. B. L. Priya, "Sugar Level Detection Using Thermal Images," International Journal of Engineering \& Technology, 2018, vol. 7, pp. 637-639, doi:10.14419/ijet.v7i4.39.25671.

18. Z. Jiang, M. Hu and G. Zhai, "Portable Health Screening Device of Respiratory Infections," 2020 IEEE International Conference on Multimedia \& Expo Workshops (ICMEW), 2020, pp. 1-2, doi: 10.1109/ICMEW46912.2020.9105969. [CrossRef]

19. S. S. Alam, T. Ahmed, M. S. Islam, M. M. F. Chowdhury, "A Smart Approach for Human Rescue and Environment Monitoring Autonomous Robot," International Journal of Mechanical Engineering and Robotics Research, Vol. 10, No. 4, pp. 209-215, April 2021. DOI: 10.18178/ijmerr.10.4.209-215. [CrossRef]

20. X. Niu, H. Han, S. Shan and X. Chen, "Continuous heart rate measurement from face: A robust rPPG approach with distribution learning," 2017 IEEE International Joint Conference on Biometrics (IJCB), 2017, pp. 642-650, doi: 10.1109/BTAS.2017.8272752. [CrossRef]

21. G. Casalino, G. Castellano and G. Zaza, "A mHealth solution for contact-less self-monitoring of blood oxygen saturation," 2020 IEEE Symposium on Computers and Communications (ISCC), 2020, pp. 1-7, doi: 10.1109/ISCC50000.2020.9219718. [CrossRef]

22. Y. Zhou, H. Ni, Q. Zhang and Q. Wu, "The Noninvasive Blood Pressure Measurement Based on Facial Images Processing," in IEEE Sensors Journal, vol. 19, no. 22, pp. 10624-10634, 15 Nov.15, 2019, doi: 10.1109/JSEN.2019.2931775. [CrossRef]

23. I Sánchez, C Vizcaya, "Tracheal and lung sounds repeatability in normal adults", Respiratory Medicine, Volume 97, Issue 12, 2003, Pages 1257-1260, ISSN 0954-6111, doi:10.1016/S0954-6111(03)00251-8. [CrossRef]

\section{AUTHORS PROFILE}

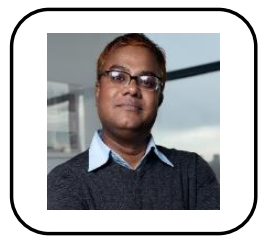

Sk. Shariful Alam, was born in Khulna, Bangladesh, in 1979. He received the B.Sc. degree in Electrical and Electronic Engineering from the Chittagong University of Engineering and Technology, Chittagong, Bangladesh, in 2003, and the M. Sc. in Telecommunications Engineering from the University of Trento, Italy, in 2010. In 2004, he joined the Department of Electronics and Communication Engineering, Khulna University of Engineering and Technology, Bangladesh, as a Lecturer. Since January 2011, he has been with the Department of Electrical, Electronic, Telecommunications Engineering, and Naval Architecture (DITEN), University of Genoa, Italy, where he has completed his PhD. His current research interests include dynamic spectrum management, spectrum sensing for wideband cognitive radios, compressive sensing, and MAC layer for cognitive radios.

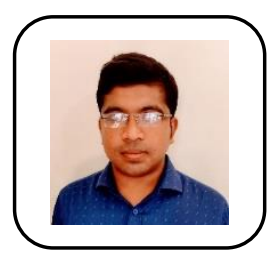

Md. Shakibul Islam, was born in Faridpur, Bangladesh, in 1998. He is currently an undergraduate student in the department of Electronics and Communication Engineering at Khulna University of Engineering \& Technology, Khulna, Bangladesh. His current areas of interest are IoT based medical equipment, EEG-based mental health monitoring, Brain computer interface,

Machine Learning, Deep Learning \& Neural Networks, and Automation.

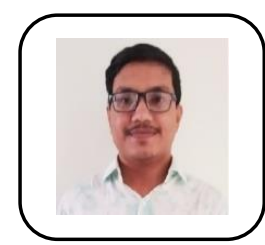

Md. Mustahsin Farhan Chowdhury, was born in Rajshahi, Bangladesh, in 1998. He is pursuing his B.Sc. degree in Electronics and Communication Engineering at Khulna University of Engineering and Technology, Khulna. Along with his major, his field of study includes automation, internet of things, Cybersecurity, and IT support. Since July Operator at Fab Lab, KUET. 2019, he has been working as a Student Lab

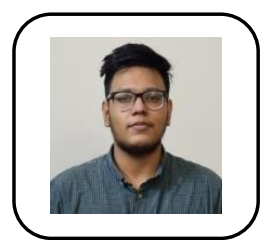

Tanim Ahmed, was born in Dhaka, Bangladesh, in 1997. He is currently pursuing his B.Sc. degree in Electronics and Communication Engineering from Khulna University of Engineering \& Technology, Khulna, Bangladesh. His current research interest includes machine learning, artificial intelligence, and image processing. 